\title{
The effectiveness of an educational project using older adult volunteers for training primary care nurse practitioners in geriatric assessment
}

\author{
Jennifer M. Hackel, Teresa M. Eliot Roberts \\ University of Massachusetts Boston, United States
}

Received: September 12, 2019

DOI: $10.5430 /$ jnep.v10n3p51
Accepted: November 18, 2019 Online Published: November 25, 2019

URL: https://doi.org/10.5430/jnep.v10n3p51

\begin{abstract}
This article reports on the effectiveness of a pilot project, where older adult volunteers attending college campus programs were recruited to act as mock patients (MP) in a two-hour clinical simulation experience for primary care nurse practitioner (NP) students learning about geriatric assessment. Primary care providers, such as NPs, study variable content on geriatrics and see older adults in their primary care clinical practica yet report they desire more time in their training to practice geriatric assessment techniques, apply clinical practice recommendations, and discuss broader aspects of cases being managed by NPs within the interdisciplinary team. Utilization of live models acting as MPs with small groups of students acting as one provider is one way in which health care trainees can take more time to learn from each other as well as the models in the simulated clinical setting. The professor wrote a hypothetical case study based on clinical practice experience that either a male or female volunteer retiree could play as the MP. The case was a 75-year-old retiree with multiple other chronic conditions, on multiple medications, presenting with acute on chronic fatigue. Of the 48 students who participated, 47 returned surveys. Aggregate scores indicated an overall effectiveness of $88 \%$ across multiple aspects of geriatric primary care. Qualitative data indicated that the NP students would like more such cases in which they get more lead time with the case information to consider the myriad factors at play and have smaller groups of students per MP. The older adults who volunteered as MPs reported overwhelmingly that they found participating in the students' education to be rewarding and a chance to offer input about improvement in the care of older adults in the current health system in our aging society. There was consistent feedback that the program should be continued and enhanced. The case content is offered in this article for use by other health care professionals who educate trainees in primary care.
\end{abstract}

Key Words: Nursing education, Elderly, Geriatric assessment, Older adult

\section{INTRODUCTION}

The population of older adults in the Unites States is expected to more than double from 46 million in 2016 to 98 million by 2060. Geriatric assessment is a critical skill for health professionals such as nurse practitioners (NPs), as older adults can present atypically, have multiple chronic conditions requiring multiple medications that may interact, and are more prone to geriatric syndromes from less wellregulated homeostatic mechanisms. ${ }^{[1]}$ Thus, there is a critical need to increase and improve the education of primary care providers (PCPs) about the vulnerabilities of older adults to decompensation from multiple interacting factors. Through incorporating live simulation into education about the unique needs of older adults in general, and geriatric assessment in

*Correspondence: Jennifer M. Hackel; Email: jennifer.hackel@umb.edu; Address: University of Massachusetts Boston, United States. 
particular, NPs can benefit from learning early on to apply these advanced skills throughout their clinical education and careers. The World Health Organization has noted that, as the growing aging population seeks primary care, our clinics and the providers should re-tool their centers to be more age-friendly and attuned to the unique challenges of older patients. ${ }^{[2]}$

Currently, graduate programs preparing NP students to be primary care providers, such as Family NPs and AdultGerontological Primary Care NPs, are not required to have more than very basic geriatric assessment content in the advanced health assessment course, where the majority of simulation occurs. Therefore, students are generally not given a chance to apply information on geriatric assessment in a simulated clinical situation, but instead learn about it in a lecture, and are expected to apply the content in their clinical placements, after the fundamental health assessment course.

Simulation has been found to be a powerful learning tool; various modes of simulation have been used with unfolding case studies to foster critical thinking where students have more time to develop these advanced skills. ${ }^{[3,4]} \mathrm{Ob}$ jective structured clinical evaluations (OSCE's) and mock patient (MP) scenarios have been found to foster reflection and deeper learning in health professional students who can talk with these "patients" and consider multiple inter-related factors without the pressure of actual clinical practice. ${ }^{[5]}$ Geriatric assessment requires that the student start with basic knowledge of (1) normal aging changes, (2) atypical disease presentation, (3) multi-morbidity, (4) geriatric pharmacology and (5) common geriatric syndromes, but many of these topics are not addressed until the NP students are seeing older adults in their internships.

A literature review on ageism in nursing education noted that both practicing nurses and nursing students exhibit ageist behaviors and attitudes. ${ }^{[6]}$ Practicing nurses' biases may come from seeing elderly patients in more acute care settings where geriatric syndromes such as frailty, delirium, incontinence and multimorbidity predominate; nursing education can significantly influence attitudes toward aging. ${ }^{[7]}$ When students engage with healthy community-dwelling older adults who can share their life experiences, and show their resiliency and adaptation to chronic conditions, this serves to counter the negative stereotypes that have been found to be prevalent in nursing students. ${ }^{[8]}$

\section{Aims}

The aims of this pilot project were to (1) assess the feasibility of live simulation in teaching geriatric assessment in an ad- vanced health assessment course, (2) assess the effectiveness of the simulation in fostering advanced gerontological assessment skills in the NP students, and (3) engage community elders in dispelling myths of aging and promoting a positive image of older adults. This experience was designed for them to see the patient in a simulated primary care setting, where understanding the patient's complaints in the context of their baseline functional level, assessing their risk for decompensation, and planning interventions to keep them out of the hospital and healthy at home as much as possible could be discussed with faculty more extensively than in the clinical setting.

\section{METHOD}

One professor created a hypothetical case study based on a real patient in her clinical practice, and wrote the script so that either a male or female volunteer older adult could play the part. The mock patient, referred to as "MP," was a 75-year-old with diabetes, hypertension and chronic musculoskeletal back and knee pain on multiple medications presenting with acutely worsening chronic fatigue and a recent fall. The script is shown in Box A (see the Appendices).

Patient actors were recruited from an educational program on campus for adults over age 50. These healthy community dwelling "elders" (most of the volunteers being in their 70 's) served to exhibit a key tenant of geriatrics: As a person ages and develops physiologic aging changes, he or she has increased vulnerability related to intercurrent acute illness in the context of multiple chronic illnesses, making them temporarily less functional and more prone to acute decompensation, hospitalization, and geriatric syndromes. ${ }^{\text {[9] }}$ However, that decompensation is not inevitable. Indeed, the primary care provider educated in geriatric practice knows the importance of awareness of altered disease presentation, early detection of alterations in function, assessment of vulnerabilities and strengths, and prevents decompensation as early as possible. ${ }^{[9]}$ The professor met with the MPs prior to class to discuss their role in the simulation prior to the class, as outlined in Box C (see the Appendices).

In preparation for the exercise, students were assigned to hear a recorded lecture which followed assigned readings, including employment of various rapid geriatric screening tools in geriatric assessment. In the recorded lecture, the students were shown an example of a patient undergoing an assessment, including the Mini-Cog ${ }^{[10]}$ and Timed Up and Go tests. ${ }^{[11]}$ At the class itself, the professor provided the students with MP's one-page "chart" (see Box B in the Appendices), including the past medical history $(\mathrm{PMH})$, medications, allergies, family and social history, code status, health care maintenance, and recent labs. The professor also pro- 
vided a "Geriatric Assessment Toolkit" booklet with various geriatric assessment tools copied from the assigned readings, which included an abbreviated Beer's Criteria list ${ }^{[12]}$ for identifying potentially inappropriate medications.

The Osher Foundation has provided funding for Life Long Learning Institutes at many universities; the professor contacted the director of her university's program and recruited the older adult through an explanatory email. She then contacted those responders who volunteered individually and asked them to come to the classroom ahead of the students to be coached in acting as MP. There, she provided volunteers with their script and explained their role in encouraging the student NPs to elicit key information in the history. She explained that they were allowed to embellish the MP's story with information unique to the volunteer, such as their personal family narratives or hobbies, social engagements and religious preferences, so long as these additions did not clash with what was being presented as "the case"- an older adult with worsening fatigue of unknown origin. The professor explained to the volunteers that the students needed to perform a thorough geriatric assessment to arrive at the correct diagnosis, which was that the "patient" in the case had developed a pneumonia, causing their fatigue, reduced involvement in usual social activities, poor appetite and higher blood sugars. The professor explained to the volunteers that, in this clinical scenario, MP experiences increasingly severe fatigue, as well as increased urination, which worsens underlying urge incontinence due to higher bladder volumes, he or she becomes more dehydrated, and presents feeling unable to perform his or her usual activities due to the underlying pneumonia. ${ }^{[13]}$

The scenario of presented by MP in the simulated visit was meant to be vague enough for students to falsely consider that the patient might have age-related fatigue - not a real diagnosis, but a common myth about aging. Alternatively, the students might consider that the patient was depressed in the context of recent widowhood, though MP volunteers were coached to not endorse depressive symptoms when the student tested them. Similarly, the physical exam performed by the students would show normal aging changes but few contributory findings for the acute fatigue, just as the patient in the real case did not have cough, fever, or adventitious respiratory findings; the real clues were in the point-of-care tests which were noted on the "chart" - borderline tachycardia and tachypnea in their vital signs, and a low oxygen saturation of $89 \%$ on room air. These tests were explained to students as having been performed by the medical assistant when the patient was placed in the exam room, as is common in primary care practice. The professor explained to the volunteers that "altered presentation" is a common challenge in geriatrics, exhibited here with the MP presenting with com-

Published by Sciedu Press plaints of worsening fatigue (which can be masked by grief in the context of recent widowhood), reduced self-care in general, and reporting recent falls, but having no significant new cough (or change in chronic cough). The professor further explained that the patient might not have lung findings on exam in the context of relative dehydration, and that a rapid pulse (which would be expected in a person with reduced oxygen saturation) might be blunted in an older adult with underlying cardiac conduction changes.

In this pilot project, six older adults volunteered to be MPs for each of the two sections of the course in which a total of a total of 49 students were enrolled and 48 attended (23 in one section and 25 in the other) which allowed for each MP to be matched with a group of four to five students. At the start of each of the two class sections, the professor introduced the six volunteers to the class, first by letting each volunteer summarize their unique lives in a few sentences - for instance one emigrated to the US from a foreign country, studied here, married, started a business, had a family, divorced and remarried, learned to fly planes, is now retired, enjoys volunteering and traveling, and is writing a book. This gave the students an appreciation for the rich lives of healthy older adults. Then the professor explained that all these volunteers were given the same script to be the mock patient, "MP."

Before breaking into groups and starting the simulation, the professor set the stage by explaining the exercise: The students were to be grouped together to share the role of one NP working in a busy primary care clinic who has had this older patient added to their schedule for chief complaint of fatigue. The professor then briefly reviewed the handouts she provided them: MP's "health record" (see Box B in the Appendices) with past medical history, medications, social history (retired teacher, widowed recently, living alone in an urban setting), recent health care maintenance and recent lab results. She pointed out MP's current vital signs and pointof-care labs, including oxygen saturation, capillary glucose, A1C and urinalysis, as provided theoretically by the medical assistant who roomed the patient. The professor then briefly reviewed what was in their booklets for the simulation entitled, "A Geriatric Assessment Mini Tool Kit" to use in assessing the patient, as had been described in the week's reading and lecture.

In order for the students to share in the steps the NP would perform in assessing the patient, and given that the groups of students were variable in size, students in each group counted off with numbers then corresponding to numbered aspects of the assessment they would perform (after first taking the history of present illness) using the tools in the mini geriatric assessment toolkit to screen the patient for: (1) 
changes in functional status, (2) depression, (3) loss of vision, (4) hearing loss, (5) nutritional concerns, (6) urinary incontinence (7) cognitive impairment, and (8) falls. The students then shared in physical assessment measures including cardiopulmonary assessment, musculoskeletal and neurologic assessment, and having the patient perform the Romberg and Timed Up and Go tests. With an average of four students per senior volunteer, this allowed students to each perform a third of the geriatric assessment. If there was time, students were encouraged to have a brief discussion with MP regarding end-of-life preferences, code status, advance directive and health proxy. The toolkit that was provided to students is too lengthy to include here, but a variety of tools are available in a review by Tatum, Talebreza and Roth ${ }^{[13]}$ or a comprehensive physical assessment or geriatrics medicine text with a chapter on geriatric assessment.

After an hour assessing their respective MPs, all the students came back together, where the professor led a brief discussion of the differential diagnosis of MP's fatigue in the context of a geriatric patient's altered disease presentation. The professor fostered critical thinking skills asking the students to explain the significance of the information they elicited about the MP's fatigue, reported falls, and objective findings such as the weight loss and low oxygen saturation. The professor asked students to discuss whether these signs would prompt them, with a real patient, to look for additional signs related to these findings (asymmetry of transmitted voice sounds in the chest), revealing that the actual patient MP's case was based on was found to have a pneumonia.

A few minutes were spent discussing MP's chronic disease self-care such as home glucose and blood pressure levels, dietary and exercise patterns, mood and medication management, which is so critical to tailoring primary care to older adults with chronic conditions. This served as a segue to having the students compare MP's meds with the Beer's List. The professor explained the evidence leading to the concept of potentially inappropriate medications (PIMs) for older adults, for instance that hypoglycemia from glyburide raises fall risk, and MP's occasional use of muscle relaxants raises the risk of falls and cognitive impairment, or that chronic use of NSAIDs can lead to GI bleeding which can cause anemia which may present as fatigue and exertional dyspnea. Thus, students were prompted to consider that finding certain medications in an older adult's medication list are an important aspect of comprehensive geriatric assessment.

Regarding assessment of the patient's reported fall, the professor reviewed the definition of falls in older adults and their significance, and the interpretation of the findings of the "Timed Up and Go" test. For the purposes of this sim- ulation, the fall was discussed as a red flag that the patient had developed a yet undiagnosed acute problem contributing to difficulty maintaining upright posture under stress, and all the forces at play in the dynamic process of ambulation. The normal test result in the simulation offered support for the conclusion that the patient did not need physical therapy at this time, but treatment of the pneumonia and supportive care to boost nutrition, hydration and maintain mobility was advisable while the patient recuperated.

Regarding cognitive status, the Mini-Cog was reviewed as a gross screen, and that further testing such as with the Montreal Cognitive Assessment ${ }^{[14]}$ would be indicated if the patient's score on the Mini-Cog was below the cutoff (under four out of five correct answers) but that a major cognitive impairment could not be diagnosed on this finding alone. It was pointed out that when memory is impaired, the patient's history may be impaired, and the input of family and/or caregivers can help NPs obtain more complete geriatric assessments.

The session was evaluated through a survey which students completed at the end of the two-hour exercise. The 12question survey used a five-point Likert scale for most of the questions in which students indicated to what degree the exercise was effective in enhancing their learning in various areas of geriatric assessment. The final two questions were open-ended for general feedback. The six older adult volunteers offered unstructured verbal feedback to the faculty after the class was dismissed regarding their perceptions of the merits or problems with the program.

\section{RESULTS}

The professors agreed the project was highly feasible as an exercise employing live simulation in teaching geriatric assessment in an advanced health assessment course, indicating achievement of the first aim, despite the multiple steps to organize the class. Had there not been an Osher Life-Long Learning Institute at the University, the professor might have had to recruit senior volunteers from a local senior center, which may have been more complicated. And, while the classroom exercise was time-consuming to create in terms of writing the script, creating the mock patient "chart" and organizing the multiple steps to take during the two-hour class, all of this has been put in the appendices (except for the compilation of geriatric screening tools for student use) for other professors to employ.

Regarding the second aim, the effectiveness of the project was ascertained through student survey analysis. Of the 48 students who participated, 47 returned surveys. Aggregate scores indicated an overall effectiveness of the program of 
$88 \%$ (see Box D in the Appendices). The most highly rated aspects of the exercise were its effectiveness in promoting students' appreciation of the richness of experience older adults have, the importance of a brief assessment of changes or deficits in basic and instrumental activities of daily living, and the importance of using the Beer's List in assessing the patient's medication list for potentially inappropriate medications. Students also frequently reported that they would have liked a chance to assess an older adult in pairs, that they wanted to practice completing a Medical Order for Life Sustaining Treatment form (MOLST), and that the exercise should continue to be offered as part of the course. Participants agreed that the third aim, engaging community elders in dispelling myths of aging and promoting a positive image of older adults, was self-evident. The senior volunteers unanimously agreed that the experience was fulfilling to them in contributing to the students' learning, while students reported the exercise promoted a positive image of aging. The senior volunteers also appreciated the chance to offer input about improvement in the care of older adults in the current health system in our aging society.

\section{DisCUSSION}

The main objective met in this exercise was increasing students' competence in geriatric assessment through application and interpretation of rapid geriatric screening tools. However, the class may have tried to accomplish too much in one two-hour exercise (see Box C-professor's role). For example, after discussing with the class that this patient could have a pneumonia, the next step is to apply a tool such as the "CRB 65" criteria, which aids in decision-making regarding whether the patient would be best served in the hospital or at home. ${ }^{[15]}$ The case was meant to be a "borderline" situation where the provider might arrive at the assessment that the patient needs admission or can be managed at home to show the value of incorporating patient preferences into care. This was the point in the exercise where the majority of the MP volunteers reported they would prefer to be treated at home if at all possible, to the great surprise of the students, who considered they all would want to be admitted if the guideline suggested this was best. This led to a brief discussion of the risks of hospitalization (iatrogenesis), the right to self-determination (medical ethics), and excess costs often related to care of older adults (health care delivery and health policy) all of which the faculty pointed out were issues to think about and discuss further in future classes. Yet it was a useful juncture to note the findings of a secondary analysis of the Health and Retirement study, which highlighted the importance of primary care providers being experts in geriatrics, and using care guidelines and quality indicators to

Published by Sciedu Press coordinate management of co-occurring chronic conditions, rather than treating each problem separately, to minimize geriatric syndromes. ${ }^{[16]}$

The majority of students noted a preference for getting the geriatric assessment content prior to the simulation, including access to the patient's "chart" and the Geriatric Assessment Toolkit, including the Beers List pocket card, a week before coming to the exercise. As an example, when the professor explained the importance of discontinuing the MP's muscle relaxant due to the risks of falls, students felt they would be better prepared to identify MP's risks with more time to consider all these factors, as they realized the gravity of the MP's polypharmacy.

Survey results confirmed that the exercise raised students' awareness of the patient's level of risk for geriatric syndromes, which predispose to disability and early mortality. ${ }^{[9]}$ MP had worsening urge incontinence, which puts a person at risk for social isolation and depression. MP was also at risk for further falls, with potentially grave consequences. The weight loss in MP's case exemplified the all too common situation of the domino effect of multiple vulnerabilities, resulting in frailty and early mortality, including the dehydrating effect of hyperglycemia which must be balanced with the risks of hypoglycemia in older adults with diabetes. ${ }^{[17]}$ Yet this was an overwhelming amount of information for students to process and would be better spread out over multiple case studies throughout their program.

The NP students gained geriatric expertise from evaluating MP's polypharmacy. At this juncture in their education, NP students are undergoing a role transition and are not yet used to taking responsibility for patients' medication programs. However, as they engaged themselves in MP's narrative, they were directed to consider the potential contribution of PIMs to MP's illness, namely, glyburide, cyclobenzaprine, and diphenhydramine, as well as dangers of multiple sources of acetaminophen with the use of over the counter "pain relievers" as well as the "sleep aid". No geriatric assessment is complete without a careful review of medications and how the older adult manages them. ${ }^{[9]}$

\section{Conclusion}

This exercise achieved its aims by first finding that the simulation was both feasible and effective. Second, student feedback and faculty observation also confirmed that the exercise fostered advanced gerontological assessment skills in the NP students. Finally, the survey results indicated that the use of live models succeeded in improving students' attitudes about aging and health. As the US health care system transitions to the Chronic Care Model from a single problem focused 
health system, ${ }^{[18]}$ PCPs with training in geriatric care will be better able to offer more comprehensive, coordinated care to our complex older patients. Live simulation employing healthy older adults as volunteers is feasible and useful in ad- vancing geriatric education, while also engendering positive attitudes toward aging.

\section{CONFlicts of INTEREST Disclosure}

The authors declare that there is no conflict of interest.

\section{REFERENCES}

[1] Kuchel GA. Aging and homeostatic regulation, IN, Halter, J.B., Ouslander, J.G., Studenski, S., High, K. Asthana, S., Ritchie, C.S. \& Supiano, M.A. (eds.) (2017). Hazzard's geriatric medicine and gerontology (7th ed.). New York, NY: McGraw Hill. 2017.

[2] World Health Organization. (2004). Towards age-friendly primary health care. Available from: https://apps . who.int/iris/bits tream/handle/10665/43030/9241592184.pdf

[3] Bowman K. The use of unfolding case studies to foster critical thinking. Journal of Nursing Education. 2017; 56: 701-702. PMid:29091243 https://doi.org/10.3928/01484834-20171 020-13

[4] Vogt MA, Schaffner BH. Evaluating interactive technology for an evolving case study on learning and satisfaction of graduate nursing students. Nurse Education in Practice. 2016; 19: 79-83. PMid:27428697 https://doi.org/10.1016/j.nepr.2016.05 .006

[5] Stanley C, Lindsay S, Parker K, et al. Value of collaboration with standardized patients and patient facilitators in enhancing reflection during the process of building a simulation. Journal of Continuing Education in the Health Professions. 2018; 38(3): 184-189. PMid:29746322 https://doi.org/10.1097/CEH.0000000000000198

[6] Gallo V. Ageism in nursing education: A review of the literature. Teaching and Learning in Nursing. 2019; 14(3): 208-215. https://doi.org/10.1016/j.teln.2019.04.004

[7] Algoso M, Peters K, Ramjan L, et al. Exploring undergraduate nursing students' perceptions of working in aged care settings: A review of the literature. Nurse Education Today. 2016; 36: 275-280. PMid:26296542 https://doi.org/10.1016/j.nedt.2015.08 .001

[8] Sarabia-Cobo CM, Pfeiffer CC. Changing negative stereotypes regarding aging in undergraduate nursing students. Nurse Education Today. 2015; 35(9): e60-e64. PMid:26116031 https://doi.org/ $10.1016 / j$.nedt .2015 .06 .006

[9] Carlson C, Merel SE, Yukawa M. Geriatric syndromes and geriatric sssessment for the generalist. Medical Clinics of North American.
2015; 99: 263-279. PMid:25700583 https://doi.org/10.1016/ j.mcna.2014.11.003

[10] Borson S, Scanlan J, Brush M, et al. The mini-cog: a cognitive 'vital signs' measure for dementia screening in multi-lingual elderly. International Journal of Geriatric Psychiatry. 2000; 15(11): 10211027. https://doi.org/10.1002/1099-1166(200011)15:11 <1021: : AID-GPS234>3.0.C0; $2-6$

[11] Browne W, Nair B. The Timed Up and Go Test. Medical Journal of Australia. 2019; 210(1): 13-14. PMid:30636313 https: //doi.org/10.5694/mja2.12045

[12] American Geriatric Society Beers Criteria Update Expert Panel. American Geriatrics Society 2019 updated AGS Beers criteria for potentially inappropriate medication use in older adults. Journal of the American Geriatrics Society. 2019; 1-21.

[13] Tatum PE, Talebreza A, Ross JS. Geriatric assessment: An officebased approach. American Family Physician. 2018; 97(12): 776-784.

[14] Nasreddine ZS, Phillips NA, Bédirian V, et al. The Montreal Cognitive Assessment, MoCA: a brief screening tool for mild cognitive impairment. Journal of the American Geriatrics Society. 2015; 53(4): 695-699. PMid:15817019 https://doi.org/10.1111/j . 1532-5415. 2005.53221.x

[15] Bauer TT, Ewig S, Marre R, et al. CRB-65 predicts death from community acquired pneumonia. Journal of Internal Medicine. 2006; 260(1): 93-101. PMid:16789984 https://doi.org/10.1111/j . 1365-2796.2006.01657.x

[16] Lee PG, Cigolle C, Blaum C. The co-occurance of chronic diseases and geriatric syndromes: The Health and Retirement study. Journal of the American Geriatrics Society. 2009; 57: 511-516. PMid:19187416 https://doi.org/10.1111/j.1532-5415.2008.02150.x

[17] American Diabetes Association. Older adults: Standards of medical care in diabetes-2018. Diabetes Care. 2018; 41(Supplement 1): S119S125. PMid:29222382 https://doi .org/10.2337/dc18-S011

[18] Davy C, Bleasel J, Liu H, et al. Effectiveness of chronic care models: opportunities for improving healthcare practice and health outcomes: A systematic review. BMC Health Services Research. 2015. 